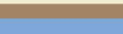

Eorberinctivit Euler

Journal of Nonlinear Mathematical Physics

\title{
Multipotentializations and nonlocal symmetries: Kupershmidt, Kaup-Kupershmidt and Sawada-Kotera equations
}

Marianna Euler, Norbert Euler, Enrique G Reyes

To cite this article: Marianna Euler, Norbert Euler, Enrique G Reyes (2017) Multipotentializations and nonlocal symmetries: Kupershmidt, Kaup-Kupershmidt and Sawada-Kotera equations, Journal of Nonlinear Mathematical Physics 24:3, 303-314, DOI: https://doi.org/10.1080/14029251.2017.1341694

To link to this article: https://doi.org/10.1080/14029251.2017.1341694

Published online: 04 January 2021 


\title{
LETTER TO THE EDITOR
}

\section{Multipotentializations and nonlocal symmetries: Kupershmidt, Kaup-Kupershmidt and Sawada-Kotera equations}

\author{
Marianna Euler ${ }^{1}$, Norbert Euler ${ }^{1}$ and Enrique G Reyes ${ }^{2}$ \\ ${ }^{1}$ Division of Mathematics, Department of Engineering Sciences and Mathematics \\ Luleå University of Technology, SE-971 87 Luleå, Sweden \\ 2 Departamento de Matemática y Ciencia de la Computación \\ Universidad de Santiago de Chile, Casilla 307 Correo 2, Santiago, Chile
}

Received 14 January 2017

Accepted 16 April 2017

\begin{abstract}
In this letter we report a new invariant for the Sawada-Kotera equation that is obtained by a systematic potentialization of the Kupershmidt equation. We show that this result can be derived from nonlocal symmetries and that, conversely, a previously known invariant of the Kaup-Kupershmidt equation can be recovered using potentializations.
\end{abstract}

Dedicated in memory to Wilhelm I Fushchich (1936-1997)

\section{Introduction}

We report a new invariant for the Sawada-Kotera equation by a systematic potentialization of the Kupershmidt equation. We deduce an invariant of the Kaup-Kupershmidt equation, obtained in [6] with the help of nonlocal symmetries. We furthermore show that the same invariant of the SawadaKotera equation can be derived by considering nonlocal symmetries.

For the benefit of clarity we first review some of the results that have been reported in [2] (see also [1] for more details on recursion operators and multipotentialization of semilinear fifth-order evolution equations).

The Kupershmidt equation ${ }^{\mathrm{a}}$

$$
K_{t}=K_{x x x x x}+\lambda\left(K_{x} K_{x x x}+K_{x x}^{2}\right)-\frac{\lambda^{2}}{5}\left(K^{2} K_{x x x}+4 K K_{x} K_{x x}+K_{x}^{3}\right)+\frac{\lambda^{4}}{125} K^{4} K_{x}
$$

( $\lambda$ is an arbitrary non-zero constant) potentializes in the so-called first potential Kupershmidt equation,

$$
U_{t}=U_{x x x x x}+\lambda U_{x x} U_{x x x}-\frac{\lambda^{2}}{5}\left(U_{x}^{2} U_{x x x}+U_{x} U_{x x}^{2}\right)+\left(\frac{\lambda}{5}\right)^{4} U_{x}^{5}
$$

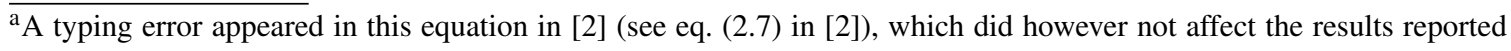
in [2] 
by the potentialization

$$
U_{x}=K
$$

Moreover, (1.1) is, by the second potentialization

$$
u_{x}=-\frac{5}{2 \lambda} e^{-2 \lambda U / 5}
$$

connected to the equation ${ }^{\mathrm{b}}$

$$
u_{t}=u_{x x x x x}-\frac{5 u_{x x} u_{x x x x}}{u_{x}}-\frac{15 u_{x x x}^{2}}{4 u_{x}}+\frac{65 u_{x x}^{2} u_{x x x}}{4 u_{x}^{2}}-\frac{135 u_{x x}^{4}}{16 u_{x}^{3}},
$$

called the second-potential Kupershmidt equation. Combining the potentializations (1.3) and (1.4), we find that (1.5) and (1.1) are related by the differential substitution

$$
K(x, t)=-\frac{5}{2 \lambda} \frac{u_{x x}}{u_{x}} .
$$

\section{Diagram 1:}

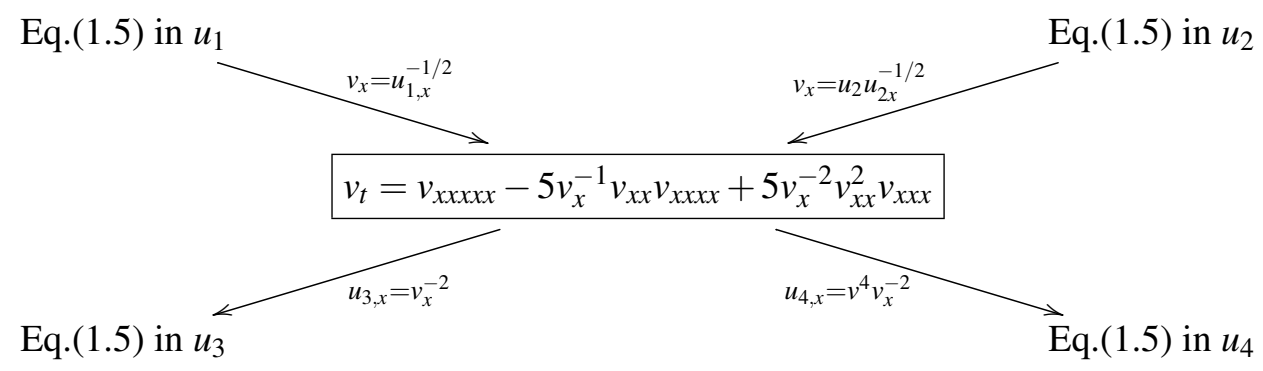

Equation (1.5) admits the following $\triangle$-auto-Bäcklund transformations that are obtained by combining potentializations as shown in Diagram 1 (see [2] for details):

$$
\begin{gathered}
u_{j+1, x x}=u_{j+1, x}\left[\frac{u_{j, x x}}{u_{j, x}}-2 \frac{u_{j, x}}{u_{j}}\right]+4 u_{j+1, x}^{3 / 4}\left[\frac{u_{j}^{1 / 2}}{u_{j, x}^{1 / 4}}\right] \\
u_{j+1, x x}=u_{j+1, x}\left[\frac{u_{j, x x}}{u_{j, x}}\right]+4 u_{j+1, x}^{3 / 4}\left[\frac{1}{u_{j, x}^{1 / 4}}\right],
\end{gathered}
$$

where $u_{j}$ and $u_{j+1}$ satisfy (1.5) for all natural numbers $j$. On the other hand, (1.5) is also related to

$$
v_{t}=v_{x x x x x}-5 \frac{v_{x x} v_{x x x x}}{v_{x}}+5 \frac{v_{x x}^{2} v_{x x x}}{v_{x}^{2}}
$$

as shown in Diagram 2.

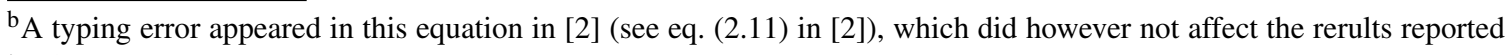
in [2] 


\section{Diagram 2:}

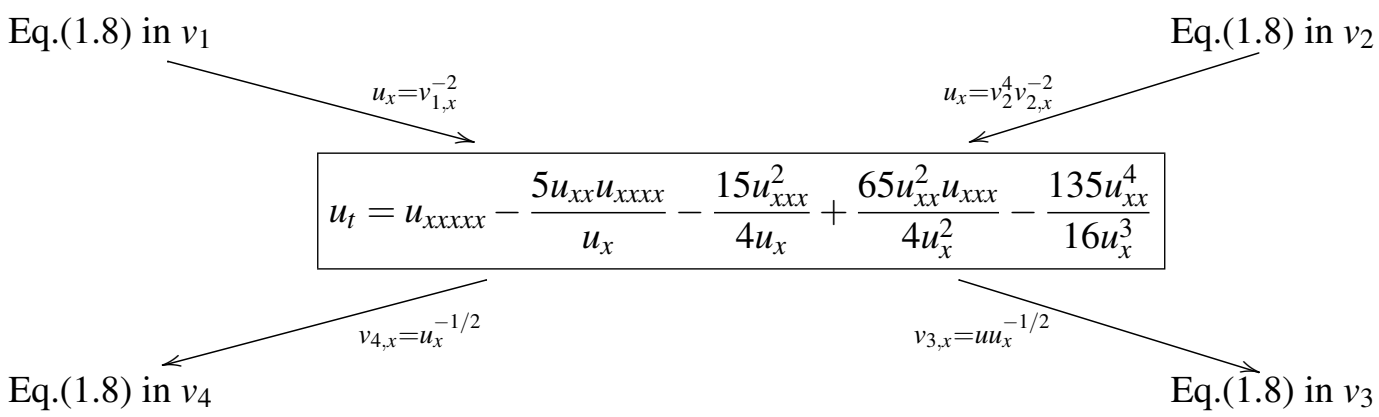

This leads to the $\triangle$-auto-Bäcklund transformations for (1.8) (see Diagram 2 and [2] for details)

$$
\begin{gathered}
v_{j+1, x x}=v_{j+1, x}\left[\frac{v_{j, x x}}{v_{j, x}}-2 \frac{v_{j, x}}{v_{j}}\right]+\frac{v_{j}^{2}}{v_{j, x}} \\
v_{j+1, x x}=v_{j+1, x}\left[\frac{v_{j, x x}}{v_{j, x}}\right]+\frac{1}{v_{j, x}} \\
v_{j+1, x x}=v_{j+1, x}\left[\frac{v_{j, x x}}{v_{j, x}}\right]-\frac{1}{v_{j, x}}
\end{gathered}
$$

where $v_{j}$ and $v_{j+1}$ satisfy (1.8) for all natural numbers $j$.

We remark that relation (1.9c) follows by applying the discrete symmetry $v \mapsto-v$ that is admitted by (1.8) to $v_{j}$ in (1.9b).

\section{Connections to the Sawada-Kotera equation}

We find that the Sawada-Kotera equation [9]

$$
S_{t}=S_{x x x x}+v S S_{x x x}+v S_{x} S_{x x}+\frac{1}{5} v^{2} S^{2} S_{x}
$$

( $v$ is an arbitrary non-zero constant) is related to the first potential Kupershmidt equation (1.2) by the differential substitution

$$
S=-\frac{\lambda}{v} U_{x x}-\frac{1}{5} \frac{\lambda^{2}}{v} U_{x}^{2} .
$$

(Note that a similar differential substitution to (2.2) was given in [4]). On the other hand, (1.2) potentializes in (1.5) by

$$
u_{x}=-\frac{5}{2} \frac{1}{\lambda} \exp \left(-\frac{2}{5} \lambda U\right)
$$

Combining these transformations, we obtain the following 
Proposition 1: The Sawada-Kotera equation (2.1) admits the solutions

$$
S(x, t)=\frac{5}{2 v}\{u, x\},
$$

where $u(x, t)$ is any non-constant solution of the second potential Kupershmidt equation (1.5) and $\{u, x\}$ is the Schwarzian derivative

$$
\{u, x\}:=\left(\frac{u_{x x}}{u_{x}}\right)_{x}-\frac{1}{2}\left(\frac{u_{x x}}{u_{x}}\right)^{2} .
$$

Moreover, relation (1.6) implies that solutions of the Sawada-Kotera equation (2.1) are

$$
S(x, t)=-\frac{\lambda}{v}\left(K_{x}+\frac{\lambda}{5} K^{2}\right),
$$

where $K(x, t)$ is any solution of the Kupershmidt equation (1.1).

The $\triangle$-auto-Bäcklund transformations (1.7a)-(1.7b) can now be applied to generate solutions for (1.5), and hence for (2.1).

We remark that relation (2.6) was previously obtained by Fordy and Gibbons [3] by factorizing a third-order linear operator.

As an example, we apply the $\triangle$-auto-Bäcklund transformation (1.7a), viz.

$$
u_{j+1, x x}=u_{j+1, x}\left[\frac{u_{j, x x}}{u_{j, x}}-2 \frac{u_{j, x}}{u_{j}}\right]+4 u_{j+1, x}^{3 / 4}\left[\frac{u_{j}^{1 / 2}}{u_{j, x}^{1 / 4}}\right] .
$$

Since (1.7a) is in the form of a Bernoulli equation in the variable $u_{j+1, x}$, we can easily integrate this equation to obtain

$$
u_{j+1, x}=\frac{u_{j, x}}{u_{j}^{2}}\left[\int\left(\frac{u_{j}}{u_{j, x}^{1 / 2}}\right) d x+c_{j}(t)\right]^{4},
$$

where $c_{j}(t)$ is an arbitrary function of $t$ that appears as a constant of integration. Inserting (2.7) with $u=u_{j+1, x}$ into (2.4), we find that $c_{j}(t)$ is an arbitrary constant.

As an explicit example, we use the following seed solution for (1.5):

$$
u_{1}(x, t)=x^{5}-180 t \text {. }
$$

Applying now relation (2.7), with $j=1$, we obtain

$$
u_{2, x}(x, t)=\left(x^{5}-180 t\right)^{-2}\left[5^{3 / 4}\left(\frac{1}{20} x^{5}+36 t\right)+c_{1} x\right]^{4} .
$$

Using $u=u_{2, x}$ given by (2.8) in relation (2.4), an explicit solution for (2.1) takes the form

$$
S(x, t)=-\frac{30\left(5 x^{8}-14400 t x^{3}-40 x^{4}+16\right)}{v\left(x^{5}+720 t+4 x\right)^{2}},
$$

where we have chosen $c_{1}=5^{-1 / 4}$ for simplicity. 
Now we obtain an invariant for the Sawada-Kotera equation (2.1). Applying the two potentializations

$$
\begin{gathered}
u_{x}=v_{x}^{-2} \\
u_{x}=v^{4} v_{x}^{-2}
\end{gathered}
$$

of (1.8), with the connection to the Sawada-Kotera equation given by (2.4), we obtain the following (see Diagram 3)

Corollary: The Sawada-Kotera equation (2.1) is invariant under the transformation $S(x, t) \mapsto$ $\bar{S}(x, t)$, in which

$$
\bar{S}(x, t)=S(x, t)+\frac{30}{v}(\ln v)_{x x}
$$

where the variables $S(x, t)$ and $v(x, t)$ are related by

$$
S(x, t)=-\frac{5}{v} \frac{v_{x x x}}{v_{x}}
$$

and $v(x, t)$ is a solution to (1.8), viz.

$$
v_{t}=v_{x x x x x}-5 \frac{v_{x x} v_{x x x x}}{v_{x}}+5 \frac{v_{x x}^{2} v_{x x x}}{v_{x}^{2}}
$$

This gives a linearization of (1.8) in terms of the Sawada-Kotera equation, namely

$$
v_{t}=v_{x x x x x}+v v_{x x} S_{x}
$$

Using any of the $\triangle$-auto-Bäcklund transformations (1.9a), (1.9b) or (1.9c), we can construct solutions of the Sawada-Kotera equation (2.1) with the above Corollary.

\section{Regarding the Kaup-Kupershmidt equation}

In this and the following section we connect the above results with nonlocal symmetries. In the paper [6], Reyes obtained an invariance transformation for the Kaup-Kupershmidt equation

$$
V_{t}=V_{x x x x x}+5 V V_{x x x}+\frac{25}{2} V_{x} V_{x x}+5 V^{2} V_{x} .
$$

In particular he reported the following 


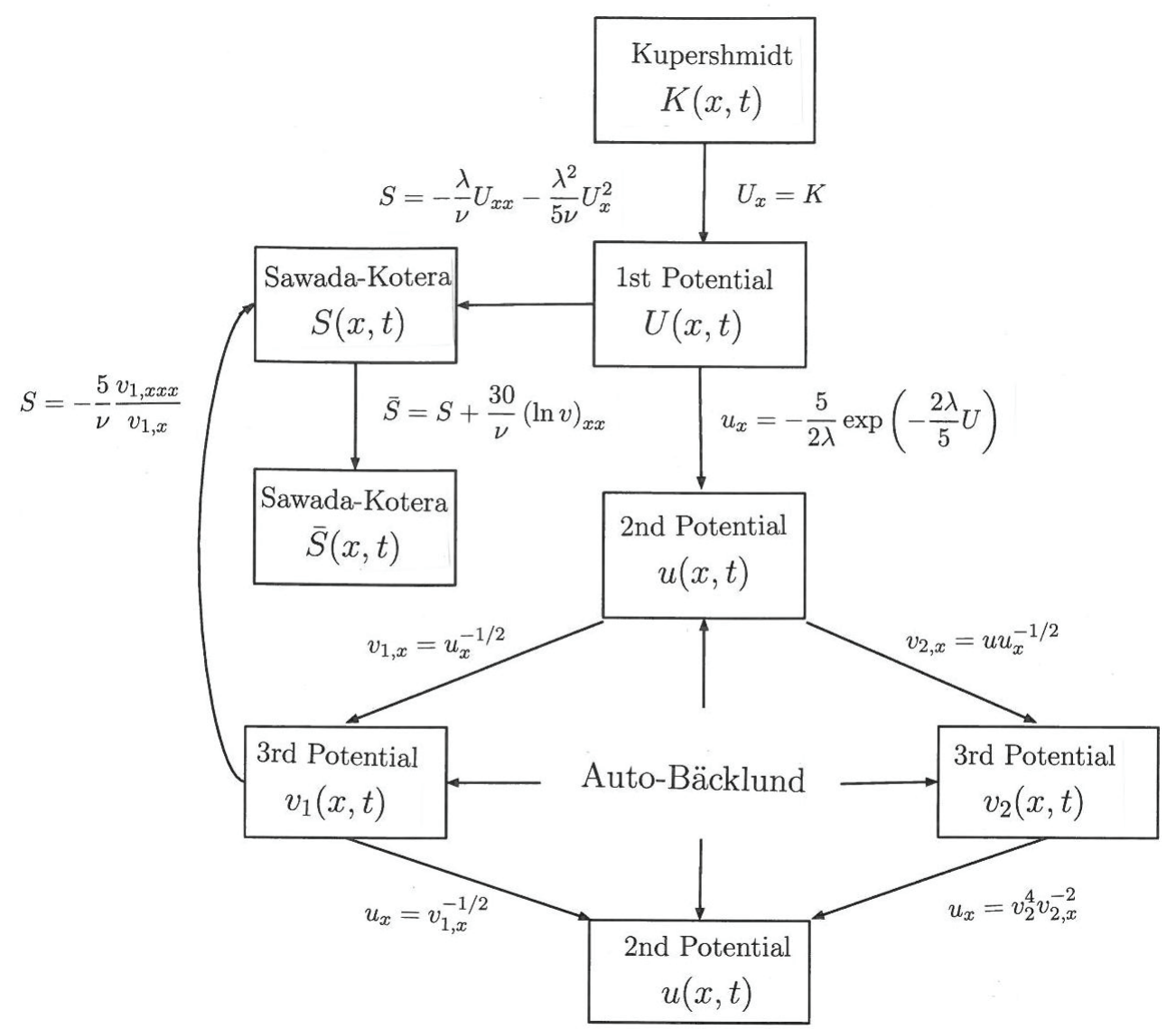

Diagram 3: An invariance transformation for the Sawada-Kotera equation (2.1)

Proposition 2: [6] The Kaup-Kupershmidt equation (3.1) is invariant under the transformation $V \mapsto \bar{V}$, in which

$$
\bar{V}=V+3(\ln u)_{x x},
$$

where the variables $V$ and $u$ are related by

$$
V(x, t)=-\frac{u_{x x x}}{u_{x}}+\frac{3}{4}\left(\frac{u_{x x}}{u_{x}}\right)^{2}
$$

and $u(x, t)$ is a solution to (1.5) viz.

$$
u_{t}=u_{x x x x x}-\frac{5 u_{x x} u_{x x x x}}{u_{x}}-\frac{15 u_{x x x}^{2}}{4 u_{x}}+\frac{65 u_{x x}^{2} u_{x x x}}{4 u_{x}^{2}}-\frac{135 u_{x x}^{4}}{16 u_{x}^{3}} .
$$

This invariance was obtained with the help of nonlocal symmetries: Equation (3.1) admits a nonlocal symmetry whose flow can be explicitly computed; consideration of this flow yields (3.2) and (3.3). We present a related computation in the next section. Now, the result given in Proposition 2 can be obtained by our multipotentialization method, namely in a similar way as was done in the 


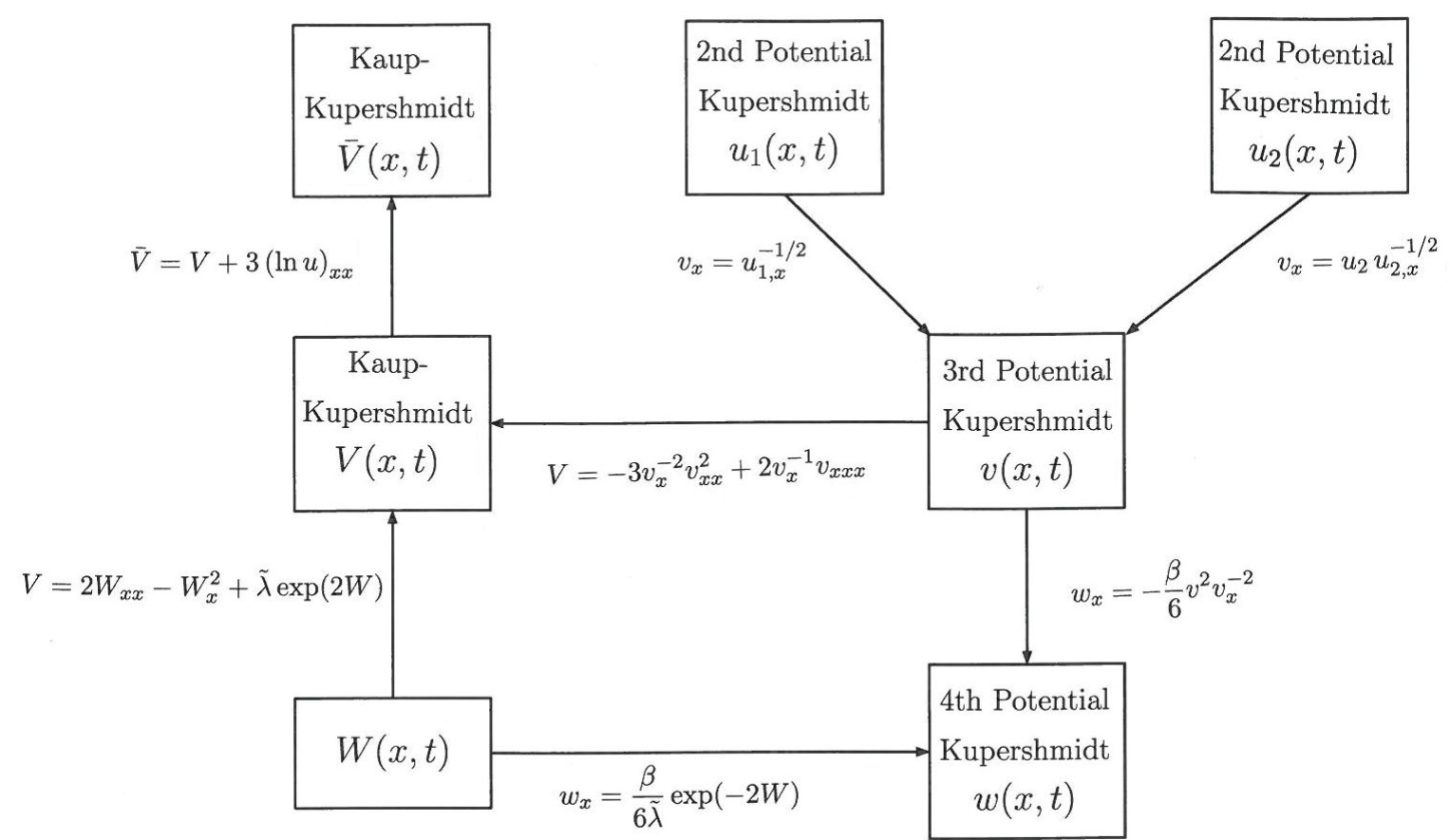

Diagram 4: An invariance transformation for the Kaup-Kupershmidt equation (3.1)

previous section for the Sawada-Kotera equation. Diagram 4 shows the connections between the equations. Besides the second potentialization of the Kupershmidt equation (1.5) (in the variables $u_{1}(x, t)$ and $\left.u_{2}(x, t)\right)$ and third potentialization of the Kupershmidt equation (1.8) (in the variable $v(x, t))$, Diagram 4 also includes a fourth potentialization of the Kupershmidt equation, namely

$$
\begin{gathered}
w_{t}=w_{x x x x x}-5 w_{x}^{-1} w_{x x} w_{x x x x}-\frac{15}{4} w_{x}^{-1} w_{x x x}^{2}+\frac{65}{4} w_{x}^{-2} w_{x x}^{2} w_{x x x}-\frac{135}{16} w_{x}^{-3} w_{x x}^{4} \\
+\frac{5 \beta}{6}\left(w_{x}^{-1} w_{x x x}-\frac{7}{4} w_{x}^{-2} w_{x x}^{2}\right)-\frac{5}{36} \beta^{2} w_{x}^{-1} .
\end{gathered}
$$

Furthermore we have the equation

$$
\begin{gathered}
W_{t}=W_{x x x x x}+5\left(W_{x x}-W_{x}^{2}+\tilde{\lambda} e^{2 W}\right) W_{x x x}-5 W_{x} W_{x x}^{2}+15 \tilde{\lambda} e^{2 W} W_{x} W_{x x} \\
+W_{x}^{5}+5 \tilde{\lambda}^{2} e^{4 W} W_{x}
\end{gathered}
$$

which is related to the fourth potential Kupershmidt equation (3.4) by a potentialization of (3.5), namely

$$
w_{x}=\frac{\beta}{6 \tilde{\lambda}} \exp (-2 W)
$$

and to the Kaup-Kupershmidt equation (3.1) by the differential substitution (given in [4])

$$
V=2 W_{x x}-W_{x}^{2}+\tilde{\lambda} \exp (-2 W) .
$$


Combining these change of variables (see Diagram 4), we obtain the differential substitution between the third potential Kupershmidt equation (1.8) and the Kaup-Kupershmidt equation (3.1), namely

$$
V(x, t)=-3 v_{x}^{-2} v_{x x}^{2}+2 v_{x}^{-1} v_{x x x}
$$

The invariance transformation given in Proposition 2 then follows (see Diagram 4).

\section{The Sawada-Kotera invariance via nonlocal symmetries}

We show that the invariance transformation (2.10) for Sawada-Kotera can be recovered with the help of nonlocal symmetries.

We replace $S$ for $(5 / v) S$ in (2.1) and we obtain the Sawada-Kotera equation in the standard form

$$
S_{t}=S_{x x x x x}+5 S S_{x x x}+5 S_{x} S_{x x}+5 S^{2} S_{x}
$$

This equation is a member of a one-parameter family of equations admitting zero curvature representations. Indeed, we recall from [7, Section 6]:

Proposition 3: [7] The family of equations

$$
S_{t}=S_{x x x x x}-\left(4 y+\frac{1}{y}\right) S S_{x x x}+5 S^{2} S_{x}-\left(2 y+\frac{3}{y}\right) S_{x} S_{x x}
$$

in which $y$ is a non-zero real parameter, is the integrability condition of the $\operatorname{sl}(2, \mathbb{R})$-valued linear problem $X \psi=\psi_{x}, T \psi=\psi_{t}$ where

$$
X=\left[\begin{array}{cc}
0 & -y / \eta^{2} \\
-\eta^{2} S & 0
\end{array}\right]
$$

and

$$
T=\left[\begin{array}{cc}
y S_{x x x}-S S_{x} & 2 y^{2} S_{x x} / \eta^{2}-y S^{2} / \eta^{2} \\
\eta^{2}\left(-S_{x x x x}+(2 y+1 / y) S S_{x x}+S_{x}^{2} / y-S^{3}\right) & -y S_{x x x}+S S_{x}
\end{array}\right]
$$

The real number $\eta$ appearing in (4.3) and (4.4) is not essential, since this "spectral parameter" can be eliminated via a simple gauge transformation. However, this linear problem does encode non-trivial information on Equation (4.2), as we will see below.

The Kaup-Kupershmidt equation corresponds to (4.2) with $y=-1 / 4$, while the Sawada-Kotera equation (4.1) is (4.2) with $y=-1$. This family contains the fifth order Korteweg-de Vries equation as well (it is enough to take $y=-1 / \sqrt{6}$ ) but we will not use this observation here. Proposition 3 allows us to find a quadratic pseudo-potential for Equation (4.2): 
Lemma: Equation (4.2) admits the quadratic pseudo-potential

$$
\begin{aligned}
\alpha_{x}= & -\eta^{2} S+\frac{y}{\eta^{2}} \alpha^{2} \\
\alpha_{t}= & -\eta^{2} S_{x x x x}+\left(2 \eta^{2} y+\frac{\eta^{2}}{y}\right) S S_{x x}+\frac{\eta^{2}}{y} S_{x}^{2}-\eta^{2} S^{3}+\left(2 S S_{x}-2 y S_{x x x}\right) \alpha \\
& +\left(\frac{y}{\eta^{2}} S^{2}-\frac{2 y^{2}}{\eta^{2}} S_{x x}\right) \alpha^{2},
\end{aligned}
$$

that is, the system (4.5a) and (4.5b) is completely integrable for $\alpha(x, t)$ whenever $S(x, t)$ is a solution to Equation (4.2).

This result generalizes some interesting computations carried out by Nucci in [5]. We write Equation (4.5b) as a conservation law, and define a corresponding potential $\delta$. We find that $\delta$ is determined by the following two compatible equations:

$$
\begin{aligned}
& \delta_{x}=\frac{2 y}{\eta^{2}} \alpha \\
& \delta_{t}=-2 y S_{x x x}+2 S S_{x}+\left(-\frac{4 y^{2}}{\eta^{2}} S_{x x}+\frac{2 y}{\eta^{2}} S^{2}\right) \alpha .
\end{aligned}
$$

We would like to find a shadow of a nonlocal symmetry for (4.2), that is, a solution to the formal linearization of (4.2) depending on $\alpha$ and $\delta$. For that, the following theorem, given in [7], where the reader can also find further references on nonlocal symmetries, is essential:

Theorem 1: [7] Consider the function

$$
G=\alpha \exp (-L(y) \delta)
$$

in which $\alpha$ and $\delta$ satisfy Equations (4.5a)-(4.6b). $G$ is the shadow of a nonlocal symmetry for Equation (4.2) if and only if

$$
L(y)=\frac{4 y^{2}+1}{10 y^{2}}
$$

and the parameter $y$ satisfies the equation

$$
-125 y^{2}\left(96 y^{6}-118 y^{4}-1+23 y^{2}\right)=0 .
$$

Since $y$ cannot be equal to zero, Equation (4.9) gives exactly six values for which (4.7) is the shadow of a nonlocal symmetry, namely

$$
y=1,-1 ; \frac{1}{4},-\frac{1}{4} ; \frac{1}{\sqrt{6}},-\frac{1}{\sqrt{6}} .
$$

The corresponding values of $L$ are $L(1)=L(-1)=1 / 2, L(1 / 4)=L(-1 / 4)=2$, and $L(1 / \sqrt{6})=$ $L(-1 / \sqrt{6})=1$. The equations obtained by replacing these values of $y$ into (4.2) are, respectively, the Sawada-Kotera, Kaup-Kupershmidt, and fifth-order KdV equations! Thus, the shadow (4.7) recognizes precisely the only 2-homogeneous polynomial evolution equations which possess an infinite number of symmetries, from a whole family of equations which are the integrability condition of overdetermined $\operatorname{sl}(2, \mathbb{R})$-valued linear problems, and which admit quadratic pseudo-potentials 
and conservation laws (the notion of $\lambda$-homogeneous equations, $\lambda \in \mathbf{R}$, and the classification cited above, appears in the paper [8] by Sanders and Wang).

We complete the shadow $G$ to a bona-fide nonlocal symmetry of the Sawada-Kotera equation following [7]:

Theorem 2: The system of equations formed by the Sawada-Kotera equation (4.1), (4.5a)-(4.6b) with $y=-1$, and the equations

$$
\beta_{x}=\frac{2 \eta^{2}}{3} \exp \left(-\frac{1}{2} \delta\right)
$$

and

$$
\beta_{t}=\frac{2 \exp \left(-\frac{\delta}{2}\right)}{3 \eta^{6}}\left[3 \eta^{6} S_{x} \alpha+\eta^{8}\left(S^{2}-S_{x x}\right)\right],
$$

admits the classical symmetry

$$
W=\alpha \exp \left(-\frac{1}{2} \delta\right) \frac{\partial}{\partial S}-\frac{\eta^{4}}{3} \exp \left(-\frac{1}{2} \delta\right) \frac{\partial}{\partial \alpha}+\beta \frac{\partial}{\partial \delta}-\frac{1}{4} \beta^{2} \frac{\partial}{\partial \beta},
$$

and therefore this vector field is a nonlocal symmetry of the Sawada-Kotera equation (4.1).

The flow of the vector field (4.12) is found by solving the system of equations

$$
\begin{aligned}
& \frac{\partial S}{\partial \tau}=\alpha \exp \left(-\frac{1}{2} \delta\right) ; \quad \frac{\partial \alpha}{\partial \tau}=\frac{-\eta^{4}}{3} \exp \left(-\frac{1}{2} \delta\right) ; \\
& \frac{\partial \delta}{\partial \tau}=\beta ; \quad \frac{\partial \beta}{\partial \tau}=-\frac{1}{4} \beta^{2}
\end{aligned}
$$

with initial conditions

$$
S(x, t, 0)=S_{0} ; \quad \alpha(x, t, 0)=\alpha_{0} ; \quad \delta(x, t, 0)=\delta_{0} ; \quad \beta(x, t, 0)=\beta_{0},
$$

in which $S_{0}, \alpha_{0}, \delta_{0}$ and $\beta_{0}$ are arbitrary particular solutions to the compatible system of equations given in Theorem 2 . The solution to this initial value problem is

$$
\begin{aligned}
& \alpha(\tau)=-\frac{4 \eta^{4} \tau}{3 \beta_{0} \tau+12} \exp \left(-\frac{1}{2} \delta\right)+\alpha_{0} \\
& \delta(\tau)=4 \ln \left|\frac{\beta_{0} \tau+4}{4}\right|+\delta_{0} \\
& \beta(\tau)=\frac{4 \beta_{0}}{\beta_{0} \tau+4} .
\end{aligned}
$$

The corresponding formula for $S(x, t, \tau)$ is obtained from the first equation in (4.13a) by using (4.15a), (4.15b) and the initial conditions above. We find the family of solutions

$$
S(x, t, \tau)=-\frac{8 \eta^{4} e^{-\delta_{0}} \tau^{2}}{3\left(\beta_{0} \tau+4\right)^{2}}+\frac{4 \tau}{\left(\beta_{0} \tau+4\right)} \alpha_{0} e^{-(1 / 2) \delta_{0}}+S_{0} .
$$


Now we remark that the foregoing analysis allows us to recover transformation (2.10). Indeed, we start from (4.16) and eliminate $\delta_{0}$ using (4.10). We obtain

$$
S=\frac{-6 \beta_{0, x}^{2} \tau^{2}}{\left(\beta_{0} \tau+4\right)^{2}}+\frac{6 \tau \beta_{0, x} \alpha_{0}}{\eta^{2}\left(\beta_{0} \tau+4\right)}+S_{0} .
$$

Now we eliminate $\alpha_{0}$ using (4.6a) [with $y=-1$ ]. We find

$$
S=\frac{-6 \beta_{0, x}^{2} \tau^{2}}{\left(\beta_{0} \tau+4\right)^{2}}-\frac{3 \tau \beta_{0, x} \delta_{0, x}}{\beta_{0} \tau+4}+S_{0} .
$$

We re-write the second summand of this expression by using the equation

$$
\beta_{0, x x}=(-1 / 2) \beta_{0, x} \delta_{0, x},
$$

which is obtained by differentiating (4.10) with respect to $x$ and simplifying the result using again (4.10). We obtain

$$
S=\frac{-6 \beta_{0, x}^{2} \tau^{2}}{\left(\beta_{0} \tau+4\right)^{2}}+\frac{6 \tau \beta_{0, x x}}{\beta_{0} \tau+4}+S_{0}
$$

or, equivalently,

$$
S=6 \frac{\partial^{2}}{\partial x^{2}} \ln (B)+S_{0}
$$

in which $B=\beta_{0} \tau+4$. This is exactly transformation (2.10).

We also recover Equation (2.11). Replacing (4.6a) [with $y=-1$ ] into Equation (4.17) we obtain

$$
\beta_{0, x x}=\frac{1}{\eta^{2}} \alpha_{0} \beta_{0, x}
$$

Differentiating (4.19) with respect to $x$ and using (4.5a) [with $y=-1$ ] we find

$$
\beta_{0, x x x}=\frac{\beta_{0, x x}}{\eta^{2}} \alpha_{0}-\beta_{0, x} S_{0}-\frac{\beta_{0, x}}{\eta^{4}} \alpha_{0}^{2} .
$$

Now we eliminate $\alpha_{0}$ from (4.20) by means of (4.19) and then we simplify the resulting expression. We obtain $S_{0}=-\beta_{0, x x x} / \beta_{0, x}$, which is equivalent to Equation (2.11) for $B$.

Finally, we consider Equation (4.11) for $\beta_{0}$. Using (4.10) we can write (4.11) as

$$
\beta_{0, t}=\frac{3 \beta_{0, x} S_{0, x} \alpha_{0}}{\eta^{2}}+\beta_{0, x} S_{0}^{2}-\beta_{0, x} S_{0, x x} .
$$

We eliminate $\alpha_{0}$ using (4.19), and we eliminate $S_{0}$ and its derivatives using the relation $S_{0}=$ $-\beta_{0, x x x} / \beta_{0, x}$ and its differential consequences. A straightforward calculation then yields

$$
\beta_{0, t}=\frac{-5 \beta_{0, x x x x} \beta_{0, x x}}{\beta_{0, x}}+\frac{5 \beta_{0, x x x} \beta_{0, x x}^{2}}{\beta_{0, x}^{2}}+\beta_{0, x x x x x} .
$$

This equation is equivalent to Equation (1.8) for $B$. 


\section{Acknowledgements}

We thank Robert Conte for useful discussions and for sharing his personal notes on the SawadaKotera and other equations. E.G.R. has been partially supported by the FONDECYT operating grant \# 1161691.

\section{References}

[1] M. Euler and N. Euler, A class of semilinear fifth-order evolution equations: recursion operators and multipotentializations, J. Nonlinear Math. Phys., 19, Suppl. 1 (2011) 61-75.

[2] M. Euler and N. Euler, Invariance of the Kaup-Kupershmidt equation and triangular auto-Bäcklund transformations, J. Nonlinear Math. Phys., 19 (2012) 1220001, 7 pp.

[3] A.P. Fordy and J. Gibbons, Some remarkable nonlinear transformations, Phys. Lett. A, 75 (1980), 325

[4] A.V. Mikhailov, A.B. Shabat and V.V. Sokolov, The symmetry approach to classification of integrable equations, in What is Integrability?, ed. E.V. Zhakarov (Springer, 1991), pp. 115-184

[5] M.C. Nucci, Pseudopotentials, Lax equations and Bäcklund transformations for non-linear evolution equations. J. Phys. A: Math. Gen. 21 (1988) 73-79.

[6] E.G. Reyes, Nonlocal symmetries and the Kaup-Kupershmidt equation, J. Math. Phys. 46, (2005) 073507, 19 pp.

[7] E.G. Reyes, Equations of Pseudo-Spherical Type (After S.S. Chern and K. Tenenblat). Results in Mathematics, 60 (2011) 53-101.

[8] J.A. Sanders and J.P. Wang, On the integrability of homogeneous scalar evolution equations. Journal of Differential Equations 147, (1998) 410-434.

[9] K Sawada and T Kotera, A Method Finding N-Soliton Solutions of the KdV and KdV-Like Equations, Progress of Theoretical Physics, 51 (1974) 1355-1367. 